\title{
Negociar en la vida cotidiana para transformar las relaciones de género: una propuesta teórica ${ }^{1}$
}

\author{
Carmen Botía Morillas \\ Universidad Pablo de Olavide. Departamento de Ciencias Sociales. Área de Sociología \\ carmenbotia@upo.es
}

Recibido: 24-07-2008

Aceptado: 16-02-2009

\section{Resumen}

Se examina la posibilidad de transformación de las relaciones de género abordando las estrategias y las prácticas de negociación puestas en juego para resolver conflictos entre géneros mediante la movilización de recursos. Se parte de los conceptos de poder de Norbert Elias y de condiciones de posibilidad como las entiende Bourdieu. Cualquier estrategia negociadora tratará de modificar el equilibrio de poder; entendido como relaciones de interdependencia en un momento y en un contexto social concreto, con posibilidad de cambiar, siendo unas condiciones de posibilidad concretas las que permitan a los géneros construidos socialmente plantear unas $u$ otras estrategias de negociación y cambio en su vida cotidiana.

Palabras clave: negociación, poder, Norbert Elias, Pierre Bourdieu, movilización de recursos.

Abstract. Negotiating in everyday life to transform gender relations: a theoretical proposal

It examines the possibility of transforming gender relations by addressing the negotiation This article examines the possibility of transforming gender relations through negotiation strategies and practices that try to solve gender conflicts by means of resource mobilization. The argument is based on two key notions: Norbert Elias' concept of power and Pierre Bourdieu's concept of conditions of possibility. Any negotiation strategy will seek to change the balance of power, understood as a (potentially changing) relation of interdependence at a specific time and social context. Some specific conditions of possibility allow socially constructed gendered agents to raise different negotiation and change strategies in their everyday lives.

Key words: negotiation, power, Norbert Elias, Pierre Bourdieu, resource mobilization.

1. El presente artículo ha partido de la reflexión teórica previa a la tesis doctoral en curso dirigida por Enrique Martín Criado, de la Universidad de Sevilla. Algunas de las ideas del artículo fueron presentadas en el Grupo 12: Sociología del Género, del IX Congreso Español de Sociología "Poder, cultura y civilización», celebrado en Barcelona los días 13 a 15 de septiembre de 2007. Agradezco a Alejandro D. Duarte Sánchez la revisión de este artículo. 


\section{Sumario}
1. Introducción
2. Conceptos de partida
5. Conclusiones
Bibliografía

4. Aproximación cualitativa

3. Consideración de las negociaciones y dos modelos de análisis

cotidianas entre los géneros como objeto

sociológico relevante

\section{Introducción}

En el presente artículo, se propone considerar como objeto de investigación relevante para la sociología las estrategias de negociación de la vida cotidiana, concretamente, las que se dan en las relaciones de pareja, estrategias que están atravesadas por las relaciones de género de manera muy importante. Se parte de la idea de que los discursos conforman y construyen la realidad que los genera, así, quienes ocupan una posición dominante en un entorno social concreto tienen más recursos y, por tanto, más capacidad de construir discursos con influencia en la configuración de las realidades de las que hablan. Se sigue la concepción de Bourdieu (1985), que afirma que los discursos como prácticas contribuyen a construir socialmente la realidad. El artículo se centra en el modo como se negocia, en las estrategias que se utilizan y en las barreras que permiten mantener o modificar los equilibrios de poder existentes en las relaciones sociales presentes en la vida cotidiana, asumiendo o cambiando así esta realidad construida mediante los discursos de quienes tienen más capacidad de influencia sobre ella. Siguiendo a Goffman (citado por Martín Criado, 1997), las interacciones sociales se generarían a partir de las acciones estratégicas que realizan los sujetos, con un sentido determinado, siguiendo cierto orden interaccional en las situaciones de la vida cotidiana.

Se entienden las relaciones de género, entre miembros de parejas heterosexuales, como de conflicto continuo, aunque muestren un aparente consenso explícito. El género influye como categoría de análisis en los comportamientos y en los asuntos sobre los que se negocia o por los que compensa trabajar estratégicamente para conseguir acuerdos beneficiosos que puedan modificar las relaciones sociales de partida.

\section{Conceptos de partida}

La movilización de recursos durante la negociación entre varones y mujeres, junto con las estrategias puestas en juego, es especialmente importante en el marco de unos discursos que deben ser entendidos como prácticas que generan realidad y que son generados fundamentalmente por el grupo dominante en el entorno social objeto de atención. Frente a la capacidad de definir la realidad, estaría la aceptación de ésta por las personas que asumen esta construcción concreta de lo cotidiano. Esto ocurre por haberse construido situaciones 
que ubican a un grupo social, las mujeres, en una posición de desventaja dentro de relaciones sociales desiguales, sin que aparentemente tengan capacidad para influir en su cambio contestándolas, ya que el hacerlo supondría el cuestionamiento de la construcción de las relaciones sociales presentes en los contextos cotidianos. Es por ello por lo que el cambio en las relaciones de género no sucede siempre y de manera continua, no obstante, el artículo pretende mostrar cómo, a partir de ciertas condiciones iniciales, el adoptar unas estrategias de negociación cuestionadoras de las relaciones sociales y de género podría hacerlo posible. Posibilidad real aunque el patriarcado no haya muerto en la actualidad, sino que ha evolucionado, asumiendo formas de control social y de control generizado mucho más sutiles y, por tanto, más efectivas y difíciles de desenmascarar, como apuntan las teorías de la diferencia de la Escuela de Milán, citadas por Biglia (2003: 2). Por ello, es necesario: «deconstruir las relaciones sociales que presentan a las mujeres como problema» (Tubert, citada por Vidal, 2002). Así, el presente artículo se centra en las relaciones de interacción entre mujeres y varones.

\section{1. "Poder» en Norbert Elias}

En este sentido, se parte de dos conceptos relacionados, el concepto de poder de Norbert Elias (1982) y el de condiciones de posibilidad tal y como lo entiende Bourdieu (1985), además del concepto de género considerado como categoría de análisis, que modula de manera muy importante a los dos anteriores. El poder en Norbert Elias (1982) implica entender las relaciones sociales como interdependientes, no cerradas ni fijas y, por tanto, con posibilidad de ser modificadas. El poder sugiere connotaciones que lo asimilan a dominación, sin embargo, para Norbert Elias tiene otra acepción, ya que en toda relación humana hay un equilibrio de poder que puede cambiar y que siempre existe allá donde exista interdependencia funcional entre sujetos sociales. El de Norbert Elias es un poder interrelacional, recíproco, interdependiente y, por tanto, un elemento integral que constituye todas las relaciones humanas. Los equilibrios de poder pueden ser más o menos fluctuantes y cambiantes.

Elias (1982) explica que el poder puede manifestarse como una relación bipolar, multipolar o en red. El autor lo ejemplifica a partir de la relación de unos padres y un bebé de un día de vida, cuando afirma que no sólo la madre y el padre mantienen una relación de poder respecto al bebé, sino que éste también la sostiene en la medida que tiene valor para éstos. Así entendido, en toda relación social existe una relación de poder o un reparto desigual de la capacidad de influir, ya que incluso en la situación anterior existiría un equilibrio de poder entendido como relación de reciprocidad, lo que no significa de equivalencia.

Desde este punto de vista, el poder es un elemento presente en todas las relaciones sociales, entendiendo así el equilibrio de poder como situación interrelacional, interdependiente y un concepto fundamental para las relaciones de género por posibilitar su cambio y una nueva construcción de las mismas. 
Así, el poder puede variar conforme se desarrolla cualquier relación social, lo cual permite mostrar el carácter de proceso que tienen las relaciones entre sujetos sociales interdependientes e inmersos en unas determinadas relaciones de poder en equilibrio coyuntural, manifestadas en un momento y en un contexto social concretos.

Dentro de esta concepción, podemos mencionar la responsabilidad de las mujeres en la gestión de desastres como ejemplos de situaciones sociales de alto riesgo y gran vulnerabilidad para sí mismas. En el tratamiento sobre la gestión de los desastres, la perspectiva teórica e investigadora ha presentando a las mujeres como víctimas y a los varones con capacidad resolutoria, cuando, en realidad, los sujetos sociales de género femenino, a pesar del fuerte impacto que los desastres tienen sobre su salud, su poder social, sus derechos humanos o su medio vital, entre otros, han puesto en marcha nuevas estrategias y se han movilizado políticamente para mitigar los fuertes impactos negativos que causaban los desastres sobre sí mismas, y así reducir los riesgos (Enarson y Meyreles, 2004). Las anteriores autoras muestran como, en situaciones tan problemáticas y difíciles que afectan tan negativamente a las mujeres, éstas movilizan sus recursos, habilidades, capacidades y conocimientos precisamente para reducir esos riesgos; y ello es relevante y pone de manifiesto la necesidad de analizar los desastres a partir de las relaciones de género puestas en juego con capacidad de movilización de recursos en las situaciones sociales concretas que les afectan.

Debe diferenciarse la definición de poder de Norbert Elias, de dominación de Weber (2002) y de dominación simbólica de Bourdieu (2000). Para Weber, el poder se entiende como dominación o la probabilidad de imponer la propia voluntad dentro de una relación social. Ésta es la acepción que aparece, por ejemplo, en el Diccionario de Sociología, poder como: «la probabilidad de que un actor, dentro de una relación social, esté en condiciones de hacer prevalecer su voluntad, incluso contra su resistencia, al margen de la base sobre la que descansa dicha probabilidad. Es decir, al margen de si ejercer ese poder es justo, es legítimo o no lo es» (Giner y Lamo de Espinosa, 1998: 578). El concepto de Weber implica un carácter intencional y voluntario de estas acciones de poder, entiende así el poder como sinónimo de dominación, aunque afirmen los anteriores autores que «el poder es relacional y nadie tiene poder en el vacío» (op. cit., 1998: 579).

Para Bourdieu (2000), sin embargo, el concepto de dominación simbólica implica obediencia en las relaciones entre mujeres y varones y, por tanto, relaciones de dominación, para unos, y de consenso, para otras, basadas en la objetividad de las estructuras sociales y de las actividades realizadas en el mercado y dentro de los hogares. El autor lo expresa del siguiente modo: «La preeminencia masculina se fundamenta en una división sexual y genérica del trabajo de producción y de reproducción biológica y social que confiere a los hombres la mejor parte, debido a los esquemas inmanentes en los hábitos sociales de mujeres y de varones. Estos esquemas o hábitos, construidos a partir de unas condiciones semejantes y, por tanto, objetivamente acordados, funcionan como universales compartidos que se imponen a cualquier agente» (Bourdieu, 2000: 49). 
Partir de los conceptos de dominación de Weber y de dominación simbóli$c a$ de Bourdieu amenaza seriamente las posibilidades de cambio. Estas acepciones no permiten abordar en su justa medida las estrategias cotidianas de las parejas que tratan de ejercerse influencia mutua y de tomar decisiones en ámbitos en los que, por sus mandatos de género, sería sustancialmente difícil que existiese la posibilidad de adoptar diferentes decisiones, acuerdos, comportamientos, roles o funciones. Aunque, ciertamente, los mandatos de género para las mujeres han ido transformándose, no ocurre del mismo modo en el caso de los varones, debido a la poca valoración que los varones, en general, otorgan a los cuidados y al tiempo dedicado a ellos, habiéndose mantenido más cercanos a sus mandatos tradicionales. Sin embargo, los mandatos de las mujeres actualmente no se corresponden exclusivamente con su vinculación a la esfera de cuidados y gestión del hogar, sino que han incorporado a su mandato la responsabilidad profesional en el marco del empleo remunerado en los momentos de su ciclo vital en los que las necesidades de cuidados de descendientes o ascendientes no son acusadas, por tanto, hay otras responsabilidades coherentes con sus mandatos de género. Puede partirse del concepto de "estructura sincrética de la condición de la mujer» de Marcela Lagarde, que generaría mujeres atrapadas por deber ser tradicionales y modernas a la vez, mujeres que deben "cuidar a los otros a la manera tradicional y, a la vez, lograr su desarrollo individual para formar parte del mundo moderno» (Lagarde, 2003: 2 y 3).

Las relaciones de poder, siguiendo con Norbert Elias (op. cit.), pueden variar en el tiempo dependiendo del aumento o la disminución de los recursos, ya sean éstos económicos, temporales, políticos, de lenguaje, etc, con los que jugar o poder ser movilizados en la práctica social cotidiana; y esto es fundamental para el análisis de las estrategias de negociación entre parejas de diferente género, ya que permite tratarlas dentro de prácticas sociales reales configuradas por relaciones de género contextualizadas.

Toda estrategia negociadora pretende modificar, y no sólo reproducir, el equilibrio de poder en favor de quien inicia el proceso negociador. De este modo, si el poder puede variar, también puede hacerlo el equilibrio de poder durante la negociación, influido éste por los distintos recursos que se controlan y pueden ser movilizados debido a que el poder es relacional y surge de la dependencia entre las partes, siendo ambas interdependientes entre sí. La necesidad mutua es la base de la relación social y ésta es también la base de las relaciones, los comportamientos y las estrategias de negociación entre los miembros de cualquier pareja, aunque nos centremos en las heterosexuales. La clave sería la posibilidad de movilización de los recursos de una parte de la relación social a otra, a pesar de partir de una distribución desigual de recursos debido a la «asignación desigual de recursos por el sistema sexo-género que sobrecarga de recursos a los varones y priva a las mujeres de los que les corresponderían» (Benhabid, 1990, citado por Cobo Bedia, 2005: 253), aunque haya que afirmar que no se trata de una privación absoluta, ya que, si no, no sería posible la movilización de recursos de uno a otro grupo. 
Entendemos la afirmación de Kate Millet (citada por Osborne, 2008), referida a que el grupo subordinado (las mujeres) recibe una ayuda insuficiente de las instituciones políticas. Esta escasez de recursos no puede llevar a afirmar que: «el grupo subordinado [...] se ve obligado a renunciar a la posibilidad de organizar una lucha y una oposición política de acuerdo con la ley» (Kate Millet, citada por Osborne y Molina, 2008: 160-161), ya que, de ser así, no sería posible movilizar los recursos para que pudieran ser trasvasados del grupo social privilegiado, los varones, con más recursos a priori, a las mujeres, grupo a priori subordinado por su escasez de recursos, que, no obstante, afirmamos, pueden ser aumentados, si se dan las condiciones de posibilidad y se siguen las estrategias de negociación adecuadas para que así sea.

En una negociación, cada una de las partes de una interacción social tratará de conseguir el mejor resultado posible; aunque esta afirmación, fruto de una lógica racional, puede no suceder automáticamente en las prácticas cotidianas. Esta realidad, modulada por relaciones de género insertas en un marco patriarcal, puede dar lugar a que las mujeres estén asumiendo situaciones desiguales generadoras de conflictos sin plantear su cambio explícitamente, bien porque no lo vean posible o porque decidan asumir su rol impuesto socialmente con tal de que impere el consenso, o el aparente consenso explícito, sin plantear ningún proceso de negociación. Así, de forma implícita, las mujeres pueden estar asumiendo muchos conflictos que probablemente no se canalicen en la relación social y que no por ello dejan de estar presentes en sus vidas, aunque no se negocie sobre ellos. Precisamente, por estar las relaciones sociales moduladas por la construcción social de los géneros, sería importante conocer cómo tienen lugar en la práctica las asunciones y los comportamientos cotidianos, e incluso cuáles de ellos podrían estar cuestionando los mandatos tradicionales de género.

\section{2. "Condiciones de posibilidad", a partir de la concepción de Pierre Bourdieu}

Evitando cierta ingenuidad, el hecho de que las relaciones sociales sean interdependientes y de que sea posible la movilización de distintos recursos que posibiliten su cambio e incluso su transformación, así como la mayor o menor influencia de una parte en la relación social, no significa que el cambio sea permanente y que la movilización de recursos ocurra continuamente. Es cierto que no todas las situaciones sociales, y quizá mucho menos, las de negociación entre géneros, pueden cambiar permanentemente en la práctica, ya que, para que éstas se modifiquen, son necesarias unas determinadas condiciones de posibilidad, entendidas como posibilidades conformadoras de relaciones sociales construidas. En palabras de Bourdieu: «la realidad es absolutamente social y las clasificaciones más "naturales" se apoyan siempre en rasgos que no tienen nada de natural y que en parte son producto de una imposición arbitraria, es decir, de un estado anterior a la relación de fuerzas en el campo de las luchas para la delimitación legítima» (Bourdieu, 1985: 89). 
Esto significa que la realidad siempre es social y se apoya en rasgos que no son naturales, sino construidos por la relación de fuerzas sociales que la generan continuamente, precisamente porque tienen capacidad para crear realidad debido a que las palabras y el lenguaje pueden imponer una nueva visión sobre lo socialmente construido o pueden contribuir a mantener la concepción preexistente. Así, deben entenderse las condiciones de posibilidad como un concepto analítico que posibilita el cambio, como elementos que lo permiten o lo propician, en definitiva, como factores que pueden favorecer o dificultar una nueva redefinición de las relaciones de género, lo que no conlleva entender estas condiciones como factores determinantes o causantes de un efecto generado o de una realidad social posterior a su existencia. Las condiciones de posibilidad serían plausibles en situaciones concretas que, a partir de Goffman (citado por Martín Criado, 1997), podemos entender como situaciones con un sentido determinado, situaciones estructuradas que ordenan las interacciones sociales que se dan en su seno. Es decir, las actividades que realizan los sujetos sociales, ni son azarosas, ni están totalmente determinadas, "corresponden al resultado de múltiples influencias en función de sus deseos, intenciones y proyectos, de sus normas de conducta, de su representación y de las actitudes y valores adquiridos», pero también dependen de las condiciones en las que viven las personas y de las oportunidades de su entorno (Lozares, Carrasquer y Domínguez, 1998: 132).

Continuando con Bourdieu y partiendo de su forma de entender las condiciones de posibilidad (Bourdieu, 1985: 96-97), se puede afirmar que es posible incidir en el mundo actuando sobre el conocimiento que de él se tiene, siendo ésta una acción que produce, impone y actúa sobre las representaciones del mundo social capaces de actuar sobre él. Así, el orden social permanece gracias a que impone esquemas de clasificación que son reconocidos. De esta manera, podrá utilizarse este concepto como una categoría de análisis relevante que permite plantear a mujeres y varones estrategias concretas y contextualizadas en sus comportamientos y prácticas cotidianas y en los discursos generados a partir de ellas, es decir, les permite negociar.

\section{3. "Género» como categoría de análisis}

Se entiende el género como categoría o esquema de interpretación de sentido estructural, del mismo modo que la clase social, que influye sobre los comportamientos, las estrategias de movilización y negociación de recursos o el manejo de conflictos para conseguir acuerdos y, con ellos, llegar a nuevos equilibrios de poder. Como punto de partida, se podría afirmar que las relaciones de género funcionan como categoría de análisis que influye en el modo como se negocian los intereses propios y los ajenos. Así, los comportamientos cotidianos en el seno de parejas heterosexuales y sus estrategias de negociación se diferencian en función del género de quien negocia fundamentalmente, aunque deban tenerse en cuenta también otros factores o categorías, como son la clase social, el nivel educativo, la profesión, la situación laboral, la edad, el hábitat, la fami- 
lia a cargo o contar con ayuda para el cuidado, entre otras, que tendrán mayor o menor relevancia en función de la relación social y del momento en el que se encuentre cada sujeto. Siguiendo a Breilh, puede afirmarse que: «La categoría género puede ser central en unos estudios o acciones específicas, pero no puede ser principal en la determinación del conjunto global del movimiento histórico de la población de la que son parte inseparable los géneros. Una cosa es la centralidad analítica y otra la condición de jerarquía principal en la determinación» (Breilh, 1994: 56). Por eso, es necesario deconstruir críticamente las relaciones de género que atraviesan a mujeres y varones, cuya identidad está también atravesada por otras categorías de análisis, que serán más o menos centrales en función de los contextos de investigación, aunque sí parece obvio que, en el caso de las interacciones entre parejas heterosexuales, las relaciones de género deben ser tratadas como prioritarias en la explicación. En esta línea, se encuentra el debate feminista (Rodríguez, 2003), el cual, posicionado entre el paradigma de la homogeneidad y el paradigma de la heterogeneidad, ofrece explicaciones enmarcadas en la complejidad, así, habría otros factores explicativos de las interacciones sociales además del género.

Debido a la existencia del patriarcado, las relaciones sociales de dominación permiten que exista un grupo, que, dentro del sistema patriarcal, son los varones, que esté investido de autoridad y legitimado por otro grupo, las mujeres, sobre el que se ejerce esa autoridad. Así, y siguiendo a Bourdieu: «los dominantes se unen entre sí con el consenso, acuerdo fundamental sobre el sentido del mundo social convertido así en mundo natural dóxico fundado en el acuerdo sobre los principios de división [...] los dominados forman parte del discurso y la conciencia [...] puesto que sólo pueden constituirse en grupo separado, movilizarse y movilizar la fuerza que detentan en estado potencial a condición de poner en tela de juicio las categorías de percepción del orden social que, siendo producto de ese orden, les imponen una actitud de reconocimiento hacia él, es decir, la sumisión» (Bourdieu, 2000: 99). De este modo, un grupo contribuiría en mayor medida a crear y construir realidad social y otro grupo tendría mayor responsabilidad en su asunción. El mismo autor lo expresa del siguiente modo: «Las categorías con arreglo a las cuales un grupo se piensa y según las cuales se representa su propia realidad contribuyen a la realidad de ese grupo» (op. cit.: 102), lo que no es sinónimo de que la realidad social en la vida cotidiana esté perfectamente determinada a priori, ya que el cambio a partir de una nueva construcción social es posible.

Hay una pregunta relevante en este sentido que se cuestiona por las razones por las que las mujeres, en las relaciones sociales entre géneros, deben ser quienes tengan que iniciar el movimiento de cambio. Biglia (2003: 16) lo plantea del siguiente modo «¿Por qué la culpa de la discriminación deben asumirla las personas discriminadas?». La autora propone que, en primer lugar, las mujeres se liberen de la culpa que el mantenimiento de relaciones desiguales comporta. Ciertamente, en cualquier situación social, si ésta es desigual, serán quienes no quieran mantener los equilibrios de poder desiguales quienes deban tratar de movilizar sus recursos para llegar a situaciones sociales menos desi- 
guales, por ello, es más difícil que el inicio del movimiento parta de quienes están en una situación más favorecida. A pesar de la posibilidad, ciertamente es difícil plantear acciones estratégicas cuestionadoras del orden patriarcal, ya que: «la ideología patriarcal está tan firmemente interiorizada, sus modos de socialización son tan perfectos que la fuerte coacción estructural en que se desarrolla la vida de las mujeres presenta para buena parte de ellas la imagen misma del comportamiento libremente deseado y elegido" (De Miguel, 2003: 132). Es complejo cuestionar la propia posición de las mujeres como grupo social, «debido a que una es mujer [...] en la medida que funciona como mujer en la estructura heterosexual dominante, y cuestionar la estructura es quizá perder algo del propio sentido» (Butler, citada por Osborne y Molina, 2008: 176). Así, es necesario coincidir con la autora respecto a que parte del problema lo constituye el concepto de "género», construido desde y contra el marco estructural patriarcal. Precisamente, el presente artículo trata de manifestar la posibilidad de este cuestionamiento, de manera colectiva evidentemente, pero también en el marco de las interacciones cotidianas en parejas formadas por varones y mujeres. Si se asume la determinación de las estructuras de manera absoluta, quedamos invalidadas para poder explicar cómo cambian de hecho las sociedades y las relaciones que se dan en su seno, concretamente, las interacciones y las relaciones de la vida cotidiana, y desde la sociología debemos dar cuenta de las posibilidades de cambio y explicarlas.

$\mathrm{Al}$ mismo tiempo, los comportamientos de varones y mujeres que viven en pareja y reproducen los mandatos de género socialmente establecidos no pueden tratarse como fenómenos privados personales, sino que son sociales y responsabilidad del conjunto colectivo, por tanto, deben convertirse en asunto público. Como acertadamente indica María Jesús Izquierdo: «Lo que sentimos, lo que somos o lo que deseamos, siendo único, se parece mucho a lo que sienten, son o desean las personas que viven circunstancias parecidas a nosotros» (Izquierdo, 2000: 26). Esta afirmación no debe significar necesariamente que los comportamientos de varones y mujeres no puedan implicar un cuestionamiento, y no una reproducción, de estos mandatos de género ${ }^{2}$. Precisamente por estar establecidos socialmente, pueden ser también socialmente cuestionados; sería clave poder conocer cómo estas prácticas de interacción social cotidianas cuestionan el comportamiento esperado para cada género dentro del marco social patriarcal en las parejas que forman.

En este marco, debe considerarse el concepto género como categoría de análisis, lo que es distinto de la consideración de género como variable estadística, entendida ésta última como una mínima unidad de análisis que permite solamente describir. Optar por el género como categoría de análisis implica remitir a un marco teórico que actúa como referencia, lo cual permite explicar la realidad social a la que tratamos de aproximarnos. Un análisis de género

2. Ya hemos visto, con Marcela Lagarde (2003), que se han sumado nuevas responsabilidades a los mandatos tradicionales de las mujeres. 
implica la incorporación sistemática de la categoría género a la hora de definir cualquier proceso, actividad o práctica ${ }^{3}$. Tanto es así, que se ha convertido en categoría irrefutable, de manera que su ausencia puede invalidar cualquier investigación que no la haya contemplado (Cobo Bedia, 2005: 256). Es necesario, además, considerarla como una categoría política forjada por el feminismo y se entiende como «ideología transformadora de la realidad» (Cobo Bedia, 2005: 257). La autora advierte, incluso, de la despolitización que afecta al concepto de género si se le desposee de su origen feminista y por tanto transformador, si, por ejemplo, se usa como sinónimo de mujer, como habitualmente sucede por el uso que los organismos internacionales hacen del concepto de género.

El género desempeña un importante papel en los comportamientos y en las negociaciones concretas de la vida cotidiana, lo que no es incompatible con asumir la diversidad social de las mujeres como una realidad con consecuencias prácticas, puesto que siguen distintas estrategias de negociación dependiendo de las posiciones sociales en las que estén ubicadas y de los recursos con los que cuenten en el presente o con los que puedan contar en el futuro y que puedan movilizar.

\section{Consideración de las negociaciones cotidianas entre los géneros como objeto sociológico relevante}

A partir de la realidad de las relaciones de género, se puede revelar cómo influyen éstas en las prácticas de la vida cotidiana, concretamente en los comportamientos y las estrategias de negociación en el seno de parejas heterosexuales. Se propone que el modo de hacerlo sea investigar tanto los procesos subjetivos o los esquemas simbólicos de los sujetos sociales que conviven, como las estructuras y los procesos objetivos o prácticos que enmarcan estos procesos de convivencia, para así poder captar las contradicciones o las transformaciones que se produzcan.

El propósito será conocer indirectamente, a partir de los discursos sobre los comportamientos, el reparto de tareas o de roles desempeñados, las prácticas de negociación de los géneros a partir del uso de los tiempos, las tareas y las responsabilidades asumidas como propias o ajenas, la forma como se establecen los «arreglos» ${ }^{4}$ o las estrategias concretas para llegar a acuerdos más ventajosos que la posición o la situación de partida. Interesa conocer las contradicciones que pueda haber, así como si se puede aceptar que el habitus (Bourdieu, 1991),

3. En palabras de Soledad Murillo: «El análisis de género es una herramienta que sirve para reconocer la repercusión que tiene para mujeres y hombres cualquier acción» (2005: 18).

4. En palabras de Teresa Torns (2005).

5. Podemos definir habitus, con las palabras de Bourdieu (1991) en El sentido práctico, del siguiente modo: «Los condicionamientos asociados a una clase particular de condiciones de existencia producen "habitus", sistemas de disposiciones duraderas y transferibles, estructuras estructurantes, es decir, principios generadores y organizadores de prácticas y repre- 
fuertemente marcado por el género, sufra desajustes de disposiciones o conviva en armonía con las prácticas. La clave sería avanzar desde un diagnóstico de necesidades de género, afectivas, laborales, temporales o de salud, entre otras, de cada miembro de las parejas heterosexuales con las que se trabaje, para, a partir de estas necesidades transformadas en intereses, conocer los distintos caminos o las distintas estrategias para satisfacerlos.

A partir de ensayos y estudios previos ${ }^{6}$, puede afirmarse que buena parte de la desigualdad en las parejas heterosexuales más cercanas al sostenimiento de relaciones igualitarias, emerge a partir del momento en el que se tienen y se cuidan a los hijos e hijas, concretamente, a partir del primero de ellos. Partiendo de esta afirmación, y asumiendo que la realidad de las relaciones de género establece desigualdades fundamentales para varones y para mujeres, al apoyarse en una diferencia construida socialmente basada en las desemejanzas biológicas de poder engendrar, gestar, parir y amamantar a una hija o a un hijo, las expectativas de ambos miembros de la pareja podrían ser muy diferentes en los momentos previos y posteriores a la llegada de la primera criatura. Probablemente, la llegada del primer descendiente es especialmente distinta para las mujeres, para quienes tiene mayor efecto la construcción social de las desigualdades por razón de género, en comparación con los varones.

El supuesto es que las mujeres irán acomodando sus expectativas familiares, profesionales o laborales, personales y vitales en función de sus prácticas reales y cotidianas y de su realidad práctica de cuidado tras la llegada de una hija o un hijo. La clave será conocer como sucede. Para el caso de los varones, sin embargo, estos cambios no serán probablemente tan relevantes, ya que, siguiendo sus mandatos de género, sus prácticas no se verán tan condicionadas con la llegada de una hija o de un hijo, como ocurre en el caso de las mujeres. Así, es más posible para los varones mantener una coherencia entre las expectativas y las prácticas previas y posteriores a la llegada de la criatura; mientras que, para las mujeres, es más difícil mantener esta coherencia y, por tanto, habría mayores diferencias prácticas entre el momento previo y posterior a la llegada del bebé, algo que evidentemente hará que modifiquen sus expectativas. $\mathrm{Si}$ esto es así, significa que la realidad está fuertemente marcada por las desiguales relaciones de género; por ello, es muy importante conocer y desvelar como

sentaciones que pueden estar objetivamente adaptadas a su fin sin suponer la búsqueda consciente de fines y el dominio expreso de las operaciones necesarias para alcanzarlos» (Bourdieu, 1991: 92). "El habitus tiende a engendrar todas las conductas "razonables" o de "sentido común" posibles dentro de los límites de estas regularidades, y sólo de éstas, que tienen todas las posibilidades de ser sancionadas positivamente porque están objetivamente ajustadas a la lógica característica de un determinado campo del que anticipan el porvenir objetivo; tiende también, al mismo tiempo, a excluir "sin violencia, sin método, sin argumentos" todas las "locuras" ("esto no es para nosotros"), es decir, todas las conductas destinadas a ser negativamente sancionadas porque son incompatibles con las condiciones objetivas" (op. cit.: 97). «Es una relación realista respecto a las cosas posibles» (op. cit.: 111).

6. Durán (1978), Izquierdo et al. (1988), Segalen (1992), Colectivo IOE (1996), Carrasco (2003) o Murillo (2006), entre otras autoras. 
funciona la realidad de género en las prácticas cotidianas de la vida en pareja ${ }^{7}$ y si estas prácticas cotidianas y las dinámicas que se dan entre ambos miembros de la pareja modifican lo que se espera de cada género.

Es decir, las prácticas influyen en las expectativas, y éstas, a su vez, en las prácticas cotidianas. Las mujeres, en el inicio de una relación de pareja, pueden desear que ésta sea igualitaria, pero si, de hecho, en la práctica, asumen el trabajo de cuidados, alimentación o gestión del hogar como propio, sus expectativas iniciales tenderán a acomodarse, con lo cual asumirán los mandatos tradicionales asociados a su género y matizando sus expectativas previas. De este modo, serán la cotidianeidad y las prácticas vividas y seguidas día a día las que moldeen las ideas sobre la propia relación y no al contrario, ya que serán las diferentes formas de resolver lo cotidiano, de compatibilizar los diferentes tiempos o de posibilitar los distintos trabajos (en los que unos cuentan y otras no con reconocimiento social y económico) los aspectos que modulen las expectativas y las aspiraciones iniciales.

El objetivo será analizar el cambio o el trabajo para la estabilidad como elementos fundamentales para explicar los comportamientos cotidianos en las parejas. Además, se pretende analizar el sentido que, especialmente las mujeres pero también los varones, otorgan a sus prácticas cotidianas y si contribuyen a modificarlas o a mantenerlas, es decir: qué luchas les interesa mantener o en qué conflictos les interesa participar y cómo lo hace cada género. Del mismo modo, es interesante conocer cuáles son los elementos diferenciales entre ambos, ya que la realidad de género incide diferencialmente en la forma como mujeres y varones satisfacen sus necesidades prácticas y sus intereses estratégicos. Respecto a las necesidades prácticas y a los intereses estratégicos, Kate Young (1991) reflexiona sobre la forma como enfrentar las necesidades de las mujeres, siendo necesario identificarlas, evaluarlas y priorizarlas para poder satisfacerlas. Caroline Moser, basándose en Maxine Molyneux (Moser, 1991: 70-71), distingue entre condición de ser mujeres y posición como mujeres, identificando la condición con los intereses prácticos. Posteriormente, se les ha llamado necesidades prácticas - condición-, frente a intereses estratégicos - posición-, determinados por su estado material diferente según el ciclo vital o la formalidad o la informalidad del trabajo. Entre las necesidades prácticas, están las derivadas de la división genérica laboral, la pobreza, la falta de educación, la capacitación, la excesiva carga de trabajo o el no acceso a la tecnología. Sin embargo, la posición como mujeres o los intereses estratégicos, que también deben tratar de satisfacerse, se derivan de una creciente toma de conciencia sobre la imposición social de las estructuras de dominio y privilegio masculino, analizando la subordinación y formulando alternativas. El objetivo final sería modificar la posición y no sólo la condición.

7. Nos centramos en parejas que se consideran «igualitarias» o de roles y funciones más equilibrados que los mantenidos en parejas con un reparto de roles tradicional. 
Es interesante, además, cuestionar teóricamente la idea que considera a las mujeres como grupo social homogéneo, ya que el hecho de considerar la categoría género como fundamental a la hora de explicar y comprender la realidad, no significa que esta categoría sea determinante e influya homogéneamente sobre todas las mujeres o sobre todos los varones del mismo modo, puesto que los sujetos sociales están atravesados por otras categorías de análisis también diferenciadoras y también fundamentales.

$\mathrm{Al}$ igual que analizar las relaciones de género en la vida cotidiana, resulta interesante estudiar también las situaciones de alejamiento y cambio o acercamiento y estabilidad al rol de género incorporado en el habitus (Bourdieu, 1991) mediante el proceso de socialización, así como la construcción social de los géneros y de las relaciones de género, tratando de poner de manifiesto las racionalidades prácticas que entran en juego. Así, se trataría de hacer explícitas también las impropiedades del rol de género en cada una de las situaciones concretas de negociación que formen parte del objeto de estudio.

$\mathrm{Al}$ mismo tiempo, las prácticas cotidianas, específicamente las prácticas cotidianas de negociación seguidas por las mujeres, no cuentan con el mismo reconocimiento social que el otorgado a las seguidas por los varones. Básicamente, la estrategia de creación de valor está asociada en mayor medida con el género femenino, y la estrategia de reclamación de valor, con el género masculino, entendiendo la creación de valor como la estrategia que se preocupa del mantenimiento de una buena relación a medio y largo plazo entre las partes que negocian y que se preocupan por satisfacer los intereses de la otra parte, a la vez que se defienden y satisfacen los propios; frente a la estrategia de reclamación de valor, que se centra en mayor medida en el beneficio a corto plazo y en la satisfacción de los intereses propios, aunque se sacrifique o se dañe la relación entre las partes a medio y largo plazo (Lax y Sebenius, 1991).

La negociación es importante, porque es una forma de gestionar y resolver los conflictos presentes en la vida cotidiana, para ello, la movilización de recursos con objeto de modificar la situación social que ha generado el conflicto necesita que compense movilizarlos y jugar socialmente con ellos, independientemente de su naturaleza, para así mejorar la situación de partida. Asumir ciertas estrategias de negociación puede tener altos costes, y poner en juego estas estrategias debe compensar, tanto si se opta por modificar la situación social de la que se parte, como si se opta por trabajar para que ésta se mantenga.

Es necesario, por tanto, establecer el marco de negociación o el marco de las negociaciones cotidianas objeto de estudio en función de las relaciones de género. Con relación a la negociación y siguiendo a Babcock y Laschever (2003), las mujeres no se atreven a pedir, por eso obtienen peores resultados en sus negociaciones, se contienen y no piden más de lo que se les ofrece y se conforman con menos de lo que necesitan o merecen. Esto se debe, para estas autoras, a que las mujeres cambian la base de comparación, y así, en lugar de compararse con los varones, se sienten afortunadas tomando como referencia de comparación a otras mujeres, sin percibir las discriminaciones 
a las que se enfrentan continuamente y que serían visibles si la referencia de comparación fuesen ellos. También es probable que, para las mujeres, sea mucho más violento que para sus compañeros plantear procesos de negociación para sí mismas, como afirma Clara Coria (1996), por lo que muchas veces ceden sin llegar a plantear o a hacer explícitas las situaciones de conflicto y asumen este conflicto implícitamente en forma de consenso, lo cual tiene importantes costes personales a largo plazo. Si no se motiva la negociación, no se explicita el conflicto y, por tanto, será más probable que se asuman relaciones sociales y de género desiguales y que se vivan situaciones sociales desiguales, sin que se cuestionen ni traten de modificarse los equilibrios de poder que las mantienen.

\section{Aproximación cualitativa y dos modelos de análisis}

Para abordar las estrategias de negociación en la vida cotidiana como elementos que pueden generar procesos de transformación de las relaciones de género tradicionales y patriarcales, la metodología cualitativa parece más adecuada. Se parte de la asunción de que las metodologías de acercamiento a la realidad social contribuyen a crear la propia realidad que pretenden medir, explicar o comprender, ya que, desde el momento en que se define la realidad o el objeto de investigación, se opta por una posición ontológica frente a ella. Así, los comportamientos y las estrategias de negociación en la vida cotidiana demandan una aproximación metodológica cualitativa, ya que permitirá acercarse a este objeto social siguiendo un método de investigación inductivo pero guiado teóricamente. Se propone partir de la construcción cualitativa de la realidad para formular las relaciones teóricas que expliquen la forma como estos procesos de cambio e incluso de transformación pueden ser posibles o como son continuamente impedidos, si fuese éste el caso.

Además, el enfoque de las investigaciones desarrolladas en este marco debería responder a una aproximación de campo, en terminología de Bourdieu, definido como: «un conjunto de relaciones históricas incorporadas a los agentes sociales» (Gutiérrez, 2002: 21); o, de manera más extensa, definido el campo como: «sistema de posiciones particular, con unas instituciones específicas y unas leyes de funcionamiento propias. Las sociedades diferenciadas estarían estructuradas en distintos campos de posiciones, con una autonomía relativa construida históricamente [...] cada campo se entendería como una estructura de posiciones con una dinámica propia en la que los agentes lucharían por apropiarse de las formas de poder específicas (capitales) [...] Esta dinámica sólo puede funcionar si los agentes ya han interiorizado previamente el interés en ellos: si en sus estructuras disposicionales están dotados de los esquemas cognitivos, apreciativos y valorativos pertinentes» (Martín Criado, 2009). «El concepto de campo es una potente herramienta para la investigación social» (Martín Criado, 2008: 29). Partiendo de este concepto de campo, el realmente útil sería el de configuración social, que puede definirse de manera similar al de campo pero con mayor propiedad en el contexto de las situaciones sociales 
cotidianas. Concepto que funcionaría también «al modo del concepto de entramado de Norbert Elias» (Martín Criado, 2008: 31).

Así, habría que optar por la metodología cualitativa para este objeto de investigación por ser más capaz de detectar lo relevante; por buscar la comprensión frente a la descripción; por interesarse por el sentido que los sujetos dan a sus acciones, y por ser interpretativa, integradora y abierta a la voz de estos sujetos o agentes sociales. En definitiva, y siguiendo a Beltrán, se optaría por la estrategia cualitativa por considerar el lenguaje como fundamental, ya que, en la cualitativa: «el lenguaje no es sólo un instrumento para investigar la sociedad, sino el objeto propio de estudio» (Beltrán, 1998: 39), y «los discursos son prácticas (y) con ellos hacemos cosas» (Goffman, citado por Martín Criado, 1997: 85).

En relación con el objeto de estudio propuesto, la perspectiva cualitativa permitirá analizar las diferentes visiones y formas de dar sentido a la realidad, integrada por comportamientos, conflictos, uso de los tiempos o estrategias de negociación en la vida cotidiana con la pareja y con el entorno. El hecho de centrarse en un análisis de la realidad discursiva no significa dejar de lado el nivel material de los procesos sociales, entendidos éstos como condiciones sociales de vida, características del trabajo remunerado y no remunerado al que pueden aspirar o expectativas laborales y personales en las que están inmersos estas mujeres y estos varones; precisamente, es con referencia a estos procesos como cada género construye su forma de dar sentido a su existencia social. La pregunta clave sería conocer cómo las prácticas o estrategias de negociación pueden modificar los equilibrios de poder, en terminología de Norbert Elias (1982), si es posible el cambio o la transformación de las relaciones de género a partir de la movilización de recursos y si se puede conocer cómo realmente están funcionando estas estrategias en los contextos sociales cotidianos.

Los modelos de análisis con los que sería posible enfrentarse a los datos construidos a partir de la metodología cualitativa serían: un modelo de análisis que incorpore la perspectiva de género y un modelo de análisis de discurso; entendiendo el análisis de discurso como práctica social contextualizada, generada a partir de unas determinadas condiciones de producción que son las que le dotan de sentido social. Se trata de concebir los discursos no con un sentido verdadero, sino como prácticas en sí mismas con sentido para quienes los emiten (Martín Criado, 1995). En definitiva, el análisis propuesto será el sociohermenéutico o vinculado al contexto social y al campo de interpretación concreto que lo haya generado, tomando los relatos o las historias personales como expresión de biografías y realidades que son sociales.

Integrar el análisis desde la perspectiva de género, sin despojarlo de su origen y razón de ser feminista, resulta fundamental, ya que supone una opción por una metodología capaz de luchar contra las inequidades y las desigualdades de género y de otra índole, debido a que las inequidades condicionan el acceso y el control a recursos y beneficios, así como a diferentes opciones vitales, ya sean éstas tiempo social, empleo, trabajo, servicios sociales, dinero, salud, expectativas sociales o expectativas vitales. Se considera a los recursos como 
fundamentales, ya que, si no se dispone de ellos, no se podrán movilizar y, por tanto, tampoco permitirán la modificación de las relaciones de género. Consecuentemente, es imprescindible realizar un análisis de género para integrar esta mirada analítica.

\section{Conclusiones}

El análisis de las negociaciones de la vida cotidiana es relevante para la sociología, por permitir la posibilidad de transformar las relaciones de género a partir del concepto de poder y equilibrio de poder de Norbert Elias (1982), en función de unas determinadas condiciones de posibilidad tal y como las entiende Bourdieu (1985), abordándolas desde una mirada cualitativa basada en la perspectiva de campo de Bourdieu (Gutiérrez, 2002 y Martín Criado, 2008) o, concretamente, desde el concepto de configuración social, con mayor propiedad en los contextos sociales cotidianos. Se plantea la temática de la negociación y las estrategias de negociación en la vida cotidiana fuera del lugar en el que estas estrategias han sido visibilizadas, el mundo de los negocios siempre dentro del ámbito público y al margen de los hogares.

El contexto de análisis se centra en las situaciones sociales cotidianas, reales para mujeres y varones, siendo fundamental centrarse en las relaciones de género en el seno de cada pareja y en el género como categoría de análisis central, y no como condición de jerarquía principal (Breilh, 1994). Integrar el análisis desde la perspectiva de género supone optar por una metodología que cuestiona las desigualdades de género y de otra índole, ya que éstas condicionan el acceso y el control a recursos y beneficios, así como a diferentes opciones vitales, ya sean éstas tiempo social, empleo, trabajo, servicios sociales, dinero, salud, expectativas sociales o expectativas vitales. La movilización de estos recursos en forma de negociación será la que permita el cambio o la estabilidad en las relaciones de género. Ésa es la potencialidad de los conceptos de poder y equilibrio de poder de Norbert Elias.

Así, se asume una importante apuesta a favor de la igualdad de oportunidades o, más específicamente, de la equidad de género, ya que la elección de los temas de investigación no es neutra. La apuesta consiste en optar por un análisis sociológico de las estrategias de negociación en la vida cotidiana dentro de las relaciones personales. Del mismo modo, se considera que la estrategia de investigación cualitativa y comprensiva es adecuada para abordar el objeto de estudio planteado.

El punto de partida es una realidad presente en las estadísticas, que muestran que hay diferencias en las prácticas de cada miembro de la pareja en función de su género. Las investigaciones derivadas del anterior planteamiento teórico pueden mostrar cómo se concretan estas diferencias en la práctica, especialmente cuando hay descendencia. Ma Ángeles Durán lo expresa del siguiente modo: «si es difícil no ser ama de casa para una mujer soltera, para una casada es casi imposible» (Durán, 1978: 19), "y una vez que llegan los hijos, la conversión de la madre en ama de casa está consumada» (op. cit.: 20), con lo 
cual son relevantes también las prácticas que se generan en el seno de estas parejas. Así, la aportación clave de la propuesta teórica sería la explicación de las dinámicas sociales que hay tras la persistencia o la transformación de las relaciones de género en las prácticas y en los contextos cotidianos.

Además, las investigaciones que puedan partir de este planteamiento teórico pueden tener consecuencias prácticas, por tratar una temática (las estrategias de negociación entre los géneros en el seno de parejas heterosexuales con descendencia) que lleva a considerar los problemas de uso de los tiempos de trabajo, familiares y personales y el tema de la corresponsabilidad entre los géneros, así como la responsabilidad del Estado y de otros agentes, como las empresas, en la inversión en servicios sociales para facilitar procesos que posibiliten que las mujeres no sean las principales responsables de la tarea de cuidado y mantenimiento de la vida humana y, por tanto, la conciliación no se centre solamente en ellas.

$\mathrm{Al}$ mismo tiempo, las investigaciones derivadas del enfoque teórico planteado pueden aportar información relevante para la toma de decisiones políticas en el marco de la Ley 3/2007, de 22 de marzo, para la igualdad efectiva de mujeres y hombres ${ }^{8}$, o incluso de la Ley Orgánica 1/2004, de 28 de diciembre, de medidas de protección integral contra la violencia de género ${ }^{9}$, así como para las leyes desarrolladas en distintas comunidades autónomas como la Ley catalana $5 / 2008^{10}$, de 24 de abril, del derecho de las mujeres a erradicar la violencia machista; o la Ley 12/2007, de 26 de noviembre, para la promoción de la igualdad de género en Andalucía ${ }^{11}$. Precisamente, aunque la primera de las leyes estatales citadas pueda equipararse con las más avanzadas de la UE, no transpone de manera fiel todas las directivas europeas en materia de igualdad de oportunidades. Como la igualdad de hecho difiere aún de la de derecho, es necesario que la investigación sociológica se sumerja en los contextos cotidianos, reales y con efectos prácticos para mujeres y para varones.

\section{Bibliografía}

BabCock, Linda y Laschever, Sara (2003). Women Don't Ask: Negotiation and the Gender Divide. Oxford: Princeton University Press.

Beltrán, Miguel (1998). "Cinco vías de acceso a la realidad social». En: GarCía FERRANDO, Manuel et al. El análisis de la realidad social. Métodos y técnicas de investigación. Madrid: Alianza. Universidad Textos, 17-45.

BigliA, Bárbara (2003). «Transformando dinámicas generizadas: propuestas de activistas de movimientos sociales mixtos». Athenea Digital, no 4, 1-25.

Bourdieu, Pierre (1985). ¿Qué significa hablar? Economia de los intercambios lingüisticos. Madrid: Akal Universitaria.

- (1991). El sentido práctico. Madrid: Taurus.

8. BOE no 71 , viernes 23 de marzo de 2007.

9. BOE no $^{\circ} 313$, de 29 de diciembre de 2004 .

10. BOE no 131 , viernes, 30 de mayo de 2008 .

11. BOJA no 247 , martes 18 de diciembre de 2007 . 
- (2000). La dominación masculina. Barcelona: Anagrama.

BREILH, Jaime (1994). «Una mirada en salud: El problema de las categorías». En: Género, poder y salud. Quito: Ibarra Ediciones, CEAS-UTM, 50-69.

CARrasCo, Cristina et al. (2003). Malabaristas de la vida: Mujeres, tiempos y trabajos. Barcelona: Icaria, Mas Madera.

Cово, Rosa (2005). «El género en las ciencias sociales». Cuadernos de Trabajo Social, vol. 18, 249-258.

COlectivo IOE (1996). Tiempo social contra reloj. Las mujeres y la transformación en los usos del tiempo. Madrid: Instituto de la Mujer.

Coria, Clara (1996). Las negociaciones nuestras de cada día. Buenos Aires: Paidós.

De Miguel, Ana (2003). «El movimiento feminista y la construcción de marcos de interpretación: el caso de la violencia de las mujeres». Revista Internacional de Sociología, 35, 127-150.

DurÁn, Ma Ángeles (1978). El ama de casa: Crítica política de la economía doméstica. Bilbao: Zero Zyx, Colección Lee y Discute, 87.

ELIAS, Norbert (1982). Sociología fundamental. Barcelona: Gedisa.

ENARSON, Elayne y MEYRELES, Lourdes (2004). «International perspectives on gender and disaster; differences and possibilities». International Journal of Sociology and Social Policy, vol. 24, 10-11, 49-93.

GINER, Salvador y LAMO DE EsPINOSA, Emilio (1998). «Poder». En: GINER, Salvador; LAMO DE EsPinOSA, Emilio y TORRES, Cristobal (eds.). Diccionario de sociología. Madrid: Alianza Editorial, 578-579.

GuTIÉRreZ, Alicia (2002). Las prácticas sociales: Una introducción a Pierre Bourdieu. Madrid: Tierra de Nadie Ediciones.

IZQUIERDO, Jesusa et al. (1988). La desigualdad de las mujeres en el uso del tiempo. Madrid: Instituto de la Mujer. Serie Estudios, 20.

IZQUIERDO, Ma Jesús (2000). Cuando los amores matan. Madrid: Ediciones Libertarias. Colección Saber Vivir.

LAGARDE, Marcela (2003). "Mujeres cuidadoras: entre la obligación y la satisfacción». En: Encuentro Anual SARE: Cuidar cuesta: Costes y beneficios del cuidado. Bilbao: Emakunde, 1-5 [en línea]. <http://www.sare-emakunde.com/media/anual/ archivosAsociados/03LAGARDE,M.-pon_Cast_1.pdf>

LAX, David y SEBENIUS, James (1991). El directivo como negociador. Madrid: Instituto de Estudios Fiscales. Ministerio de Economía y Hacienda.

Lozares, Carlos; Carrasquer, Pilar y Domínguez, Marius (1998). "Las representaciones en el mundo de la vida cotidiana». Papers. Revista de Sociología, 55 , 131-149.

MARTÍN CRIADO, Enrique (1995). «Propuestas de método para evitar la deriva interpretativa en la producción y análisis sociológico de discursos». Comunicación presentada en el $V$ Congreso Español de Sociología. Granada, 28-30 de septiembre.

- (1997). «El grupo de discusión como situación social». Revista Española de Investigaciones Sociológicas, 79, 81-110.

- (2008). «El concepto de campo como herramienta metodológica». Revista Española de Investigaciones Sociológicas, 123, 11-34.

- (2009). «Habitus». En: REYES, Román (dir.). Diccionario crítico de ciencias sociales: terminología cientifico-social, tomo 2. Madrid: Plaza y Valdés.

Moser, Caroline (1991). «La planificación de género en el tercer mundo: enfrentando las necesidades practicas y estratégicas de género». En: GUZMÁN, Virginia et al. Una nueva lectura: Género en el desarrollo. Lima: Ediciones Flora Tristán, 55-124. 
MuriLlo, Soledad (2005). «El tiempo de trabajo y el tiempo personal, un conflicto de intereses». En: Formación y acreditación en consultoría para la igualdad de mujeres y hombres. Bilbao: Emakunde (formato $\mathrm{CD}$ ).

- (2006). El mito de la vida privada: de la entrega al tiempo privado. Madrid: Siglo XXI.

Osborne, Raquel y Molina, Cristina (2008). «Evolución del concepto de género. (Selección de textos de Beauvoir, Millet, Rubin y Butler)». Empiria: Revista de Metodologia de Ciencias Sociales, 15, 147-182.

RodrígueZ, Lidia (2003). «Género y discurso: Acercamiento teórico crítico al discurso de las mujeres». Trayectorias, vol. 5, 12, 34-44.

SEgalen, Martine (1992). Antropologia histórica de la familia. Madrid: Taurus.

TORNS, Teresa (2005). «De la imposible conciliación a los permanentes malos arreglos». Cuadernos de Relaciones Laborales, 23, 15-33.

VIDAL, Rafael (2002). «Discurso feminista y temporalidad: la descomposición postmoderna de las identidades de género». Especulo. Revista de Estudios Literarios, 20 [en línea]. <http://www.ucm.es/info/especulo/numero20/dis_femi.html>.

Weber, Max (2002). Economía y sociedad. México: Fondo de Cultura Económica. YounG, Kate (1991). "Reflexiones sobre como enfrentar las necesidades de las mujeres». En: GUZMÁN, Virginia et al. Una nueva lectura: género en el desarrollo. Lima: Ediciones Flora Tristán, 55-124. 